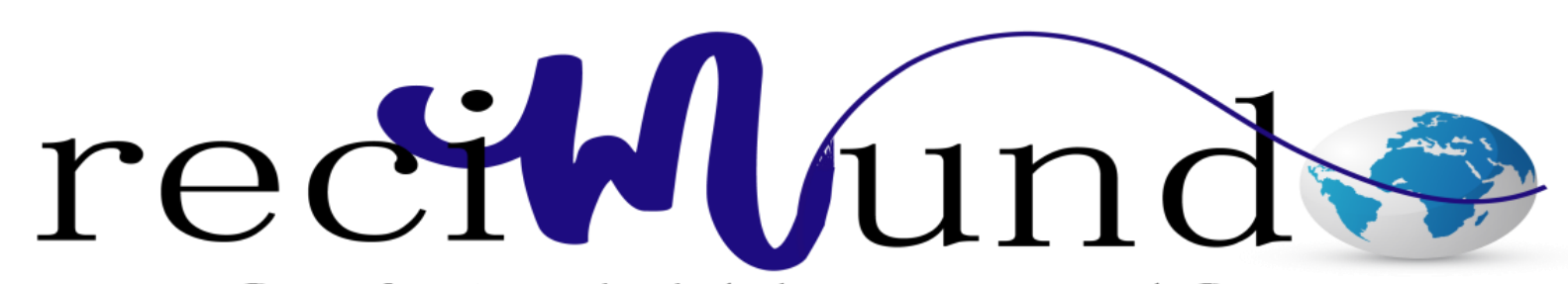

Revista Científica Mundo de la Investigación y el Conocimiento

Erick Hugo Zambrano Franco ${ }^{\text {a; }}$ Kevin Antony Zambrano Franco ${ }^{\text {b; }}$ José Alejandro Zambrano Moreira ${ }^{c}$; Susan Stefania Toral Fiallos ${ }^{\mathrm{d}}$

Etiología y tratamiento de la dispareumia

Etiology and treatment of dyspareumia

Revista Científica Mundo de la Investigación y el Conocimiento. Vol. 3 núm.3. Esp., noviembre, ISSN: 2588-073X, 2019, pp. 643-658

DOI: $10.26820 /$ recimundo/3.(3.Esp).noviembre.2019.643-658

URL: http://recimundo.com/index.php/es/article/view/617

Código UNESCO: 3205 Medicina Interna

Tipo de Investigación: Artículo de Revisión

(C) RECIMUNDO; Editorial Saberes del Conocimiento, 2019

Recibido: 15/09/2019

Aceptado: 23/10/2019

Publicado: 30/11/2019

Correspondencia: $\underline{\text { dr_ezambrano@hotmail.com }}$

a. Médico; Investigador Independiente; Guayaquil, Ecuador; dr_ezambrano@ hotmail.com

b. Médico; Investigador Independiente; Guayaquil, Ecuador; kazfzamb@gmail.com

c. Médico; Investigador Independiente; Guayaquil, Ecuador; azm_92@hotmail.com

d. Médica; Investigadora Independiente; Guayaquil, Ecuador; susan_toral01@ @otmail.com 


\section{Etiología y tratamiento de la dispareumia}

Vol. 3, núm. 3 Esp., (2019)

Erick Hugo Zambrano Franco; Kevin Antony Zambrano Franco; José Alejandro Zambrano Moreira; Susan Stefania Toral Fiallos

\section{RESUMEN}

Este trabajo investigativo presenta información sobre las disfunciones sexuales del sexo femenino y se trató de forma específica a la dispareunia, su etiología, factores psicológicos y fisiológicos y tratamientos para esta. Mediante el desarrollo de un diseño bibliográfico y una metodología de revisión, se efectuó búsqueda y revisión documental de literatura científico académica determinada, la cual fue extraída de diversas bases de datos. Se concluyó principalmente que, en el estudio de los casos de dispareunia, se es necesario realizar un tratamiento donde cada paciente se evalué fisiológicamente y psicológicamente, para detectar cualquier influencia traumática a la hora de describir sus sensaciones. De forma secundaria, se observa la importancia de aumentar la formación de las personas en el placer y satisfacción sexual desde jóvenes.

Palabras clave: Disfunciones; Etiología; Fisiológicos; Tratamiento; Sensaciones. 
Etiología y tratamiento de la dispareumia

Vol. 3, núm. 3 Esp., (2019)

Erick Hugo Zambrano Franco; Kevin Antony Zambrano Franco; José Alejandro Zambrano

Moreira; Susan Stefania Toral Fiallos

\begin{abstract}
This research paper presents information on the sexual dysfunctions of the female sex and the dyspareunia, its etiology, psychological and physiological factors and treatments for this were specifically treated. Through the development of a bibliographic design and a review methodology, a search and documentary review of determined academic scientific literature was carried out, which was extracted from various databases. It was mainly concluded that, in the study of cases of dyspareunia, it is necessary to perform a treatment where each patient was evaluated physiologically and psychologically, to detect any traumatic influence when describing their feelings. In a secondary way, the importance of increasing the training of people in sexual pleasure and satisfaction from young people is observed.
\end{abstract}

Keywords: Dysfunctions; Etiology; Physiological; Treatment; Sensations. 


\section{Etiología y tratamiento de la dispareumia}

Vol. 3, núm. 3 Esp., (2019)

Erick Hugo Zambrano Franco; Kevin Antony Zambrano Franco; José Alejandro Zambrano Moreira; Susan Stefania Toral Fiallos

\section{Introducción.}

Las disfunciones sexuales son definidas como alteraciones en algunos de los procesos del ciclo de respuesta sexual o dolor asociado a la relación sexual (Perez, 2014). En el siguiente artículo se presenta primeramente introducción a las disfunciones sexuales del sexo femenino, para luego tratar de forma específica a la dispareunia, su etiología, los factores fisiológicos, psicológicos y los tratamientos conocidos en la actualidad para tratarla

Becerra-Alfonso (2015) indica que el sexo es un influenciador del bienestar imprescindible para la vida, razón por la cual el ejercicio saludable y satisfactorio del mismo ha impulsado un importante aumento en las investigaciones de la sexualidad femenina en las últimas dos décadas, pero que debido a la multifactorialidad que posee cada mujer en sus respuesta sexuales, ha llevado a que los estudios deban de tomar en cuenta el desarrollo y percepción sociocultural de cada país. Dando como ejemplo que en América Latina el fomento del "sexo seguro" se ha limitado simplemente al cuidado y prevención de enfermedades transmisibles, del embarazo precoz y se ha dejado completamente de lado la implantación de programas que promuevan el placer y satisfacción sexual, limitando la disponibilidad de información que existe relacionada a las disfunciones sexuales en América Latina.

Como resultado de esto, la información disponible sobre trastornos sexuales femeninos es muy limitada y la investigación sobre el efecto de estos desórdenes en la calidad de vida de las mujeres es virtualmente inexistente (García \& Harlow, 2010).

La aparente falta de interés en los trastornos sexuales de parte de los programas de salud reproductiva opera como una barrera para que las mujeres hablen abiertamente de su sexualidad 


\section{Etiología y tratamiento de la dispareumia}

Vol. 3, núm. 3 Esp., (2019)

Erick Hugo Zambrano Franco; Kevin Antony Zambrano Franco; José Alejandro Zambrano Moreira; Susan Stefania Toral Fiallos

con los proveedores de salud. Lo anterior explica parcialmente por qué los programas de salud reproductiva se enfocan sólo en las "consecuencias" que acompañan el ejercicio de la sexualidad, como el embarazo y las enfermedades de transmisión sexual, y no promueven el tamizaje o escrutinio de los trastornos sexuales. (García \& Harlow, 2010).

Sixto, Ruiz, \& Blanco (2004) indican que, a lo largo del ciclo vital, es común presentar algún tipo de disfunción sexual que, lógicamente, va a incidir en la calidad de vida, y apuntan que los estudios recientes han dado manifiesto que todas las disfunciones alteran la calidad de vida, no sólo repercutiendo en el paciente, sino que también se va a encontrar afectada la pareja $y$, por ende, el entorno familiar y social.

De la misma manera explican que las dificultades sexuales en personas mayores pueden iniciar desde una etapa temprana su vida sexual, pueden haberse desarrollado a través del tiempo, o simplemente aparecer repentinamente impidiendo total o de forma parcial participar en las etapas del acto sexual.

Para clasificar las disfunciones sexuales estos tratadistas usan el Manual diagnóstico y estadístico de los trastornos mentales que usa la siguiente clasificación:

- Trastornos del deseo sexual

- $\quad$ Trastornos de la excitación sexual

- Trastornos orgásmicos

- $\quad$ Trastornos sexuales por dolor

- $\quad$ Otras disfunciones sexuales 


\section{Etiología y tratamiento de la dispareumia}

Vol. 3, núm. 3 Esp., (2019)

Erick Hugo Zambrano Franco; Kevin Antony Zambrano Franco; José Alejandro Zambrano Moreira; Susan Stefania Toral Fiallos

La Dispareunia, entra en la clasificación de Trastornos Sexuales por dolor, la cual tiene por significado "coito doloroso, difícil o malo". Decimos que existe dispareunia cuando existe dolor persistente o recurrente en genitales durante la relación coital. Aunque hablemos de «dolor» son igualmente válidos síntomas y expresiones como escozor, desgarro, ardor o quemazón, picor o desagrado. (Sixto, Ruiz, \& Blanco, 2004).

Esta se puede presentar al inicio de la penetración, en algún punto de la entrada de la vagina, o sentirse más profundamente. La intensidad y duración de estas sensaciones son igualmente variables, abarcando desde experiencias ligeras y breves hasta persistentes y de gran intensidad (Perez, 2014).

Aunque sea común que una disfunción sexual como la dispareunia en la mayoría de la ocasión se origine de un problema orgánicos, su diagnóstico se puede llevar a cabo de forma independiente a su etiología y solo se hace necesario saber de su existencia. (Becerra, 2015).

Las causas que originan a padecer dispareunia pueden ser diversas, tal como se dijo con anterioridad, por lo que conviene hacer una investigación en la cual se puedan identificar los diversos factores que pueden existir, y con esto, decidir qué tipos de tratamientos pueden ser los más eficaces dependiendo de cada caso en estudio.

\section{Materiales y Métodos.}

Las herramientas y materiales de trabajo que se conciben para el desarrollo de este trabajo investigativo son los siguientes: computadores personales con conexión a internet y un cúmulo de contenidos científico académicos diversos, con los que se persigue aportar información de 


\section{Etiología y tratamiento de la dispareumia}

Vol. 3, núm. 3 Esp., (2019)

Erick Hugo Zambrano Franco; Kevin Antony Zambrano Franco; José Alejandro Zambrano Moreira; Susan Stefania Toral Fiallos

calidad y actualizada respecto a etiología y tratamiento de la dispareunia. Se escoge un diseño documental, de tipo no experimental, a nivel descriptivo, de corte transeccional y bajo una metodología inductiva y de revisión.

Esta investigación se enfoca en la búsqueda y revisión documental de literatura científico académica seleccionada, que por una parte, está disponible determinadas bases de datos, entre las que figuran: ResearchGate, PubMed, SciELO, Redalyc, Scientific Direct y entre otras; y por la otra, mediante el uso del material físicamente disponible en la biblioteca universitaria como en otras particulares, representando todo ello parte esencial del proceso investigativo y de comprensión que facilita la síntesis de la mejor evidencia disponible, y a su vez representando todo ello uno de los resultados esperados.

Principalmente se realiza una búsqueda aleatoria y consecutiva en las mencionadas bases de datos, usando las expresiones "etiología de la dispareunia", "tratamiento de la dispareunia", "factores influyentes en la dispareunia" y "síntomas de la dispaurenia", lo que aproximadamente resultó en más de un centenar de miles de registros bibliográficos. Luego éstos se filtran en base a criterios de: idioma español, relevancia, correlación temática y fecha de publicación en los últimos catorce años, sin descartar por tipo de material bibliográfico; es decir, se escogen títulos de artículos científicos, ensayos, revisiones sistemáticas, protocolos, editoriales, libros, boletines, folletos, tesis de grado, posgrado y doctorado, noticias científicas, entre otros documentos e información de interés científico y académico.

Se desestimaron estudios de series de casos y reportes de casos; artículos de revisión narrativa; editoriales, y cartas al editor. 


\section{Etiología y tratamiento de la dispareumia}

Vol. 3, núm. 3 Esp., (2019)

Erick Hugo Zambrano Franco; Kevin Antony Zambrano Franco; José Alejandro Zambrano Moreira; Susan Stefania Toral Fiallos

Seguidamente, se efectúa la selección y clasificación de la literatura científico académica físicamente disponible, aplicando, en términos generales, los criterios antes mencionados, siendo a partir de allí que el equipo investigador le da la correspondiente lectura crítica y análisis de toda esa evidencia científica, lo que resultó consecutiva y consensuadamente en el fundamento de las ideas y planteamientos aquí plasmados.

\section{Resultados.}

La Dispareunia, puede ser multifactorial, tanto factores físicos como psicológicos pueden contribuir a su aparición. Las posibles causas incluyen cambios hormonales, diversas afecciones médicas o nerviosas y problemas emocionales como ansiedad o depresión. Una cosa puede desencadenar fácilmente una cascada de problemas (Harvard Health Publishing, 2019).

Se ha asociado con una actitud más negativa hacia la sexualidad, con un mayor deterioro de la función sexual y con niveles más bajos de ajuste de la relación. Las mujeres con dispareunia tienen una frecuencia más baja de relaciones sexuales y niveles más bajos de deseo y excitación, menos sensación orgásmica con la estimulación oral y las relaciones sexuales. (Heim, 2015).

Algunas personas la sufren en las primeras ocasiones que tienen relaciones, pero en otros casos aparece transcurrido un tiempo. Dentro de las causas más frecuentes, se tiene la sequedad o falta de líquido para humedecer la zona vaginal, que puede ser originado por tener relaciones con poca excitación, cambios hormonales de la menopausia o algún medicamento (Sociedad Española de Medicina de Familia Y Comunitaria, 2013). 


\section{Etiología y tratamiento de la dispareumia}

Vol. 3, núm. 3 Esp., (2019)

Erick Hugo Zambrano Franco; Kevin Antony Zambrano Franco; José Alejandro Zambrano Moreira; Susan Stefania Toral Fiallos

Debido a que existen numerosas condiciones físicas que pueden contribuir al dolor durante los encuentros sexuales, siempre se indica un examen físico cuidadoso y un historial médico con tales quejas. Las causas físicas comunes de molestias coitales incluyen infecciones de la vagina, el tracto urinario inferior, el cuello uterino o las trompas de Falopio, clamidia, tricomonas, bacterias coliformes); endometriosis tejido cicatricial, quistes y tumores de ovarios. (Bionity, s.f.).

Pérez (2010) enlista los factores que pueden motivar la aparición de la Dispareunia de la siguiente forma:

\section{Causas Biológicas}

- Superficial-introital o medio vaginal, o ambas.

- Infecciosas: Vulvitis, vestibulitis vulgar, vaginitis, cistitis

- Inflamatorias: con regulación de las células mastoideas.

- Hormonales: atrofia bulbo vaginal.

- Anatómicas: himen fibroso, himen cribiforme, agenesia vaginal, síndrome de Rokitansky.

- Musculares: primaria o secundaria a hiperactividad del elevador del músculo anal.

- Iatrogénicas: mala evolución de cirugía genital o perineal, radioterapia pélvica

- Neurológicas: inclusive dolor neuropático.

- Inmunológicas y del tejido conectivo (síndrome de Sjögren).

- Vasculares.

- Comorbilidad con otras disfunciones sexuales que pueden originar dispareunia crónica con alteraciones en las fases de deseo, excitación y orgasmo. 


\section{Etiología y tratamiento de la dispareumia}

Vol. 3, núm. 3 Esp., (2019)

Erick Hugo Zambrano Franco; Kevin Antony Zambrano Franco; José Alejandro Zambrano Moreira; Susan Stefania Toral Fiallos

- Dispareunia profunda

- Endometriosis

- Enfermedad pélvica inflamatoria.

- Varicocele pélvico.

- Dolor pélvico crónico y dolor referido

- Posterior a radioterapia pélvica o endovaginal.

- Síndrome de atrapamiento del nervio cutáneo abdominal (ACNES).

\section{Psicosexual}

- Comorbilidad con alteraciones en el deseo, excitación o vaginismo.

- Violación o abuso sexual en el pasado.

- Desórdenes afectivos: depresión y ansiedad.

- Catastrofismo como principal modalidad psicológica.

\section{Contexto relacionado con la pareja}

- Problemas con la intimidad emocional.

- Preámbulo inadecuado.

- Conflictos de pareja: verbales, físicos o pareja sexualmente abusiva.

- Poca compatibilidad anatómica (tamaño del pene o genitales femeninos infantiles).

- Insatisfacción sexual y, consecuentemente, excitación inadecuada. 


\section{Etiología y tratamiento de la dispareumia}

Vol. 3, núm. 3 Esp., (2019)

Erick Hugo Zambrano Franco; Kevin Antony Zambrano Franco; José Alejandro Zambrano Moreira; Susan Stefania Toral Fiallos

El tratamiento de la dispareunia debe estar enfocado al diagnóstico etiológico y existen múltiples tratamientos sintomáticos y terapias entre los cuales encontramos:

1. Terapia sexual: consiste en la educación o reeducación sexual de la paciente.

2. Tratamiento farmacológico: fármacos utilizados en la dispareunia, gabapentina, pregabalina, IRSNs (duloxetina), antidepresivos tricíclicos, BZD, relajantes musculares, anticolinérgicos.

3. Tratamientos tópicos vaginales:

- Lubricantes vaginales: Pueden ser a base de agua, aceite, glicerina o silicona.

- Estrógenos vaginales: Mejoran la atrofia vaginal, el prurito y la dispareunia, ya que normalizan el $\mathrm{pH}$ ácido, aumentan la vascularización del epitelio, aumentan la respuesta lubrificadora y disminuyen la sequedad vaginal

- El uso de estrógenos orales sólo debería utilizarse si existe sintomatología climatérica importante asociada y sin contraindicación a la TH.

- Hidratantes vaginales: reemplazan las secreciones vaginales normales. (Alejandra, Teresa, Noelia, \& Irene).

Sobre los factores psicológicos, Becerra-Alfonso (2015) expone ciertos estudios donde se ha demostrado que estos pueden condicionar la localización del dolor sexual; se han reportado una mayor incidencia de vulvodinia en mujeres con historial de abuso sexual y físico en la infancia, además de la relación entre depresión y ansiedad en la aparición de dolor vulvar crónico y dispareunia. Esta, además indica que las experiencias que pueden experimentarse a la hora de la penetración pueden tener repercusiones en un futuro, generando sensaciones de temor que 


\section{Etiología y tratamiento de la dispareumia}

Vol. 3, núm. 3 Esp., (2019)

Erick Hugo Zambrano Franco; Kevin Antony Zambrano Franco; José Alejandro Zambrano Moreira; Susan Stefania Toral Fiallos

inhiben la excitación genital, reduciendo la lubricación, lo cual termina generando un círculo vicioso de "miedo-dolor".

Los temores a la sexualidad detectados se originan por el conflicto entre el deseo y la prohibición sexual, lo que lleva a la incapacidad de ejercer satisfactoriamente su sexualidad. En estas disfunciones sexuales se encuentra un tipo específico de frustración, pues el deseo, la excitación y el orgasmo están presentes, pero el coito no se puede realizar de manera satisfactoria. (Claudia, Jorge, Norma, \& Bertha, 2010).

Factores psicológicos o emocionales pueden estar involucrados. El estrés, la ansiedad, la depresión, la culpa, una historia de abuso sexual, un examen pélvico molesto en el pasado o problemas de relación también pueden ser la raíz del dolor sexual. (Harvard Health Publishing, 2019).

Sixto, Ruiz, \& Blanco (2004), enlistan los siguientes factores psicológicos que pueden ser causa de Dispareunia:

I. Factores educacionales:

- La ingenuidad sexual y el desconocimiento de la respuesta sexual

- Mala información generador de miedo y ansiedad

- Visión negativa de la sexualidad

- Fobia a la penetración

II. Factores relacionales:

- Relación de pareja en conflictos 


\section{Etiología y tratamiento de la dispareumia}

Vol. 3, núm. 3 Esp., (2019)

Erick Hugo Zambrano Franco; Kevin Antony Zambrano Franco; José Alejandro Zambrano Moreira; Susan Stefania Toral Fiallos

\section{- $\quad$ Déficit de excitación}

III. Otras causas de origen sexual

- Deseo sexual inhibido

Sobre los tratamientos psicológicos y la efectividad, Francisco \& Crespo (2001) indicaban de la falta de investigación enfocada de forma metodológica que constatara la eficiencia de los tratamientos psicológicos para esta disfunción. Este tipo de disfunción usualmente estaría relacionado de forma secundario a algún problema probablemente de tipo físico. Lo que ha generado que la forma más común de tratarla, sea con tratamiento médicos o quirúrgicos, pero, no obstante, incluso cuando se origina de un factor físico, se va a generar una intensa respuesta psicológica que será necesaria reducir de alguna manera. Muchas de las mujeres tratadas con esos procedimientos usualmente necesitaran una terapia sexual o tratamiento cognitivo conductual para disipar cualquier respuesta psicológica no deseada a la hora del coito.

En un estudio llevado a cabo por López (2008), se hayo como resultado, que existe un gran componente psicológico y psicosexual relacionado con la dispareunia. Dando como recomendación realizar exámenes de forma periódica, prohibir el coito de inicio, los juegos sexuales sin penetración, pasar del dolor a no dolor, buscar sensación agradables y orgasmo, realizar actividades de gimnasia para la relajación y rehabilitación del suelo pélvico, trabajar con la pareja en tratamientos psicosexuales, quitar los miedos y expresar los sentimientos.

El tratamiento deberá ser llevado por etapas, respetando el ritmo cada paciente, hasta que haya desaparecido el dolor durante la penetración. Se considera de gran utilidad la participación 


\section{Etiología y tratamiento de la dispareumia}

Vol. 3, núm. 3 Esp., (2019)

Erick Hugo Zambrano Franco; Kevin Antony Zambrano Franco; José Alejandro Zambrano Moreira; Susan Stefania Toral Fiallos

de su pareja desde un lugar de comprensión, sostén y acompañamiento activo durante todo el proceso (Rosana \& Cedrés, 2019).

\section{Conclusiones.}

La etiología de la dispareunia puede ser multifactorial, razón por la cual llevar a cabo una investigación a profundidad por cada paciente, es muy importante. No solo hay que enfocarse en los factores físicos que pudieron haber influenciado, sino también hacer una revisión al estado psicológico del paciente, para descartar cualquier influencia traumática a la hora de describir sus sensaciones. Esta revisión debe de ser llevada de forma cuidadosa, sin intimidar, ya que esto puede alterar sus respuestas y por lo tanto dañar el diagnostico.

De la misma forma, se observa la importancia de aumentar la formación sobre el tema desde la temprana edad por parte de las instituciones encargadas de la enseñanza, Una de las mejores formas de reducir las disfunciones sexuales, es formando a cada una de las personas en el placer y satisfacción sexual, aumentando la información que existe relacionada ello, para que los jóvenes sean cocientes de lo que está sucediendo en su cuerpo y actúen con responsabilidad.

\section{Bibliografía.}

Alejandra, R. C., Teresa, B. A., Noelia, J. M., \& Irene, P. S. (s.f.). Dispareunia. Obtenido de Asociacion Abadia Zumaque: https://www.abadiazumaque.com/docs/guias/073.pdf

Becerra, Y. A. (2015). Trastornos del dolor sexual femenino: una revisión de su definición, etiología y prevalencia. MÉD UIS, 3(28), 267-272.
Bionity.
(s.f.).
Dyspareunia.
Obtenido
de Bionity: https://www.bionity.com/en/encyclopedia/Dyspareunia.html\#Symptoms_in_women 


\section{Etiología y tratamiento de la dispareumia}

Vol. 3, núm. 3 Esp., (2019)

Erick Hugo Zambrano Franco; Kevin Antony Zambrano Franco; José Alejandro Zambrano

Moreira; Susan Stefania Toral Fiallos

Claudia, S. B., Jorge, C. M., Norma, C. A., \& Bertha, T. R. (2010). Perfiles e indicadores psicológicos relacionados con la dispareunia y el vaginismo: Estudio cualitativo. Segunda parte. Salud Mental, 33(5), 437-449.

Francisco, J. L., \& Crespo, M. (2001). Tratamientos psicológicos eficaces para las disfunciones sexuales. Psicothema, 13(3), 428-441.

García, H., \& Harlow, S. D. (2010). Cuando el coito produce dolor: una exploración de la sexualidad femenina en el noroeste de México. Salud Pública de México, 52(2), 148-155.

Harvard Health Publishing. (25 de Octubre de 2019). When sex gives more pain than pleasure. Obtenido de Harvard Health Publishing: https://www.health.harvard.edu/pain/when-sexgives-more-pain-than-pleasure

Heim, L. (15 de Abril de 2015). Evaluation and Differential Diagnosis of Dyspareunia. Amerycan Family Physician, 63(8), 1535-1545.

López, O. (Octubre de 2008). Dispareunia: investigación de causa física y de causa infecciosa crónica (estudio prospectivo de 4 años). Elsevier, 35(5), 152-159.

Perez, G. (Diciembre de 2014). Abordaje integral del dolor coital tratamiento en un caso de dispareunia. Enfermería: Cuidados humanizados, 3(2), 22-28.

Pérez, M. C. (2010). Disfunciones sexuales femeninas: transtornos dolorosos sexuales. Ginecol Obstet Mex, 78, 527-548.

Rosana, P., \& Cedrés, S. (24 de Octubre de 2019). Disfunciones sexuales femeninas. Obtenido de Plenus: Centro Medico Sexologico: http://www.plenus.com.uy/docs/mujer/Disfunciones\%20sexuales\%20femeninas.pdf

Sixto, A. V., Ruiz, L. G.-G., \& Blanco, C. S. (2004). Guía de buena práctica clínica en disfunciones sexuales. Madrid: International Marketing \& Communications.

Sociedad Española de Medicina de Familia Y Comunitaria. (2013). Dispareunia. Obtenido de semFYC: https://www.semfyc.es/wp-content/uploads/2016/07/06_06.pdf 
Etiología y tratamiento de la dispareumia

Vol. 3, núm. 3 Esp., (2019)

Erick Hugo Zambrano Franco; Kevin Antony Zambrano Franco; José Alejandro Zambrano Moreira; Susan Stefania Toral Fiallos

$$
\text { (c) }
$$

\section{RECONOCIMIENTO-NOCOMERCIAL-COMPARTIRIGUAL}

CC BY-NC-SA

ESTA LICENCIA PERMITE A OTROS ENTREMEZCLAR, AJUSTAR Y CONSTRUIR A PARTIR DE SU OBRA CON FINES NO COMERCIALES, SIEMPRE Y CUANDO LE RECONOZCAN LA AUTORÍA Y SUS NUEVAS CREACIONES ESTÉN BAJO UNA LICENCIA CON LOS MISMOS TÉRMINOS. 